Pedagogías de
la lengua 


\title{
Enunciación
}

\section{Evolución del repertorio didáctico durante la formación inicial del profesorado en Letras}

\author{
Evolution of the didactic repertoire during the initial training of language teachers
}

\author{
Marcos Maldonado*
}

\section{Resumen}

Nos proponemos en este artículo abordar la problemática de la evolución del repertorio didáctico (Cicurel, 2016) durante las prácticas de enseñanza en la formación inicial del profesorado en lengua. Mediante el estudio de caso (Stake, 1999), interpretamos las trazas discursivas del proceso de enseñanza/aprendizaje que se encuentran en los guiones conjeturales y en los autorregistros, dispositivos de formación, de una estudiante del profesorado en Letras de la Universidad de Buenos Aires, Argentina. Nuestros datos de análisis son documentos escritos recolectados del Campus Virtual de la Cátedra Didáctica Especial y Prácticas de la Enseñanza. Debido a la naturaleza discursiva y dialógica de los datos, nuestra metodología se construye desde el análisis interpretativo hermenéutico del discurso en interacción y del discurso de la acción. Mediante la alternancia de posturas inductivas y deductivas intentamos obtener indicios que nos permitan dar respuesta a nuestra problemática de interés: por qué y cómo evoluciona el repertorio didáctico de los estudiantes en formación durante la realización de sus prácticas profesionales. Los resultados del análisis de las interacciones verbales entre la formadora y la practicante nos demuestran que la utilización de determinados gestos discursivos permite crear situaciones significativas que potencializan el aprendizaje y producen una movilización cognitiva que motiva la evolución del repertorio didáctico.

Palabras clave: repertorio didáctico evolutivo, interacción didáctica, resignificación, acción pedagógica, dispositivos de formación, análisis discursivo.

\begin{abstract}
In this paper we propose to address the problematic of the evolution of the didactic repertoire (Cicurel, 2016) during the teaching training in the initial training of teachers in language. Through the case study (Stake, 1999), we interpret the discursive traces of the teaching-learning process found in the guiones conjeturales and in the autorregistros, training devices, of a student of the language teaching of the University of Buenos Aires, Argentine. Our analysis data are written documents collected from the Virtual Campus of the Chair of Special Didactics and Teaching Practices. Due to the discursive and dialogical nature of the data, the methodology employed is constructed from the interpretive-hermeneutical analysis of the discourse-in-interaction and the discourse of action. Through the alternation of inductive and deductive postures we try to get evidence that allows us to respond to our problematic of interest: why and how the didactic repertoire of students in training evolves during their professional practices? The results obtained show us that the use of certain discursive strategies allows us to create significant situations that potentiate learning and produce a cognitive mobilization in the intern that motivates the evolution of the didactic repertoire.

Keywords: evolutionary didactic repertoire, didactic interaction, resignification, pedagogical action, training instruments, discursive analysis. * Doctor en Didáctica de la Lengua y la Literatura por la Universitat de Barcelona. Miembro asociado al laboratorio DILTEC, Université Sorbonne-Nouvelle Paris 3,
Francia, investigador del grupo TALES (Théorie, Action, Langage et Savoirs) de la Universidad de Ginebra, Suiza, y colaborador científico de la Facultad de Humanidades de la Universidad Nacional de Catamarca, Argentina. Correo electrónico: profmarcosmaldonado@gmail.com
\end{abstract}

Cómo citar: Maldonado, M. (2019). Evolución del repertorio didáctico durante la formación inicial del profesorado en Letras. Enunciación, 25(1), 62-75. DOI: http:// doi.org/10.14483/22486798.15325

Artículo postulado: 16 de septiembre de 2019; aprobado: 07 de noviembre de 2019. 


\section{Introducción}

Las prácticas de enseñanza en la formación inicial funcionan como una bisagra en la construcción del perfil docente de un profesor, puesto que es el momento en el que, por lo general, los estudiantes de las licenciaturas se ponen por primera vez al frente a un grupo de alumnos. Es cuando comienzan a asumir ciertas responsabilidades propias de la profesión (Edelstein y Coria, 1995). A pesar del sentido de iniciación con el cual se asocia esta instancia de formación, los estudiantes no son ajenos ni tampoco novatos en este tipo de prácticas sociales. Cuando llegan a este punto, detrás de ellos hay una experiencia pedagógica y sociocultural vivida, ya sea como protagonistas o como testigos, que les ha permitido construir determinadas representaciones o imágenes sobre el acto de enseñar lengua y literatura. De esta trayectoria personal o historia pedagógica han emergido saberes o esquemas de acción (Perrenoud, 2012) propios de la docencia. Para Cicurel (2002) y Causa (2012), este conjunto de saberes construidos biográficamente conforma el repertorio didáctico $(\mathrm{RD})$ de los practicantes; una estructura cognitiva heterogénea, subjetiva y dinámica que les permite tomar decisiones y actuar en función de la situación didáctica en la que se encuentran.

La idea de un RD conformado implica un cambio de perspectiva en los modelos de formación inicial. Los estudiantes en formación no son sujetos vacíos de saberes pedagógicos, sino que poseen todo un sistema de saberes consolidados que va a funcionar como punto de referencia para sus prácticas y para la asimilación de nuevos saberes. Desde esta perspectiva, la problemática de la adquisición de saberes profesionales durante las prácticas de enseñanza reposa en la expansión o evolución del RD: ¿Cómo y por qué evoluciona? ¿Qué gestos pedagógicos estimulan esta evolución? ¿Cómo lograr que los practicantes modifiquen ciertas estructuras o esquemas de acción contenidos en sus RD? Las respuestas a estas preguntas facilitarían, en primer lugar, pensar en un modelo de formación que pone como protagonistas a los mismos sujetos de esta, desde su complejidad e historicidad (Cambra y Palou, 2007), y, por el otro, diseñar estrategias didácticas que se orienten a provocar cambios significativos en la estructuración del RD de los practicantes.

Con el fin de aproximarnos a una respuesta a esta problemática, nos proponemos en este artículo observar el proceso de las prácticas de una estudiante, Laura, del profesorado en Letras de la Universidad de Buenos Aires y las interacciones que tiene con su formadora, Martina, en relación con sus prácticas. Ante este caso, nos hacemos la siguiente pregunta: ¿Qué gestos didácticos utiliza Martina para crear situaciones significativas y potenciales de aprendizaje que permiten una evolución del repertorio didáctico? Para poder responderla, analizaremos el dispositivo de formación, los guiones conjeturales y los autorregistros (Bombini, 2012, 2018), que Laura y Martina utilizan para trabajar sobre las prácticas, en busca de trazas del proceso de enseñanza/aprendizaje que las interactuantes dejan en sus discursos. Estas trazas, en tanto dépot laisse' à l'occasion d'un processus tal y como las define Leleu-Merviel (2013, p. 67), nos ofrecen indicios que nos permiten explicar y comprender los eventos didácticos ocurridos en la interacción verbal.

Nuestra exposición está organizada en tres momentos. En primer lugar, nos referimos a nuestro marco teórico en torno a la complejidad del campo de las prácticas de enseñanza en la formación inicial del profesorado y a la configuración y conformación del repertorio didáctico. Luego, nos focalizamos en la contextualización de la investigación y en la descripción de nuestra metodología de análisis. Por último, exponemos los resultados de nuestro análisis, que nos Ilevan a ofrecer una respuesta a nuestras preguntas de investigación. 


\section{Marco teórico}

\section{El doble objeto del sistema de actividad de las prácticas de enseñanza}

En nuestra convivencia social, asumimos roles determinados según el lugar que ocupamos dentro de la estructura social y de la interacción que sostenemos con otros sujetos. Nos definimos por la presencia de estos y actuamos en consecuencia, guiados por nuestros intereses. Construimos los sentidos de nuestras acciones, según A. Schütz (2008), de la tensión entre el aquí del yo y el allí del otro. Para E. Goffman (1973), la interacción cara a cara es definida como la influencia recíproca de un individuo sobre las acciones del otro.

La actividad colectiva durante la resolución de una tarea común puede llevar la interacción a una negociación progresiva en la que los sujetos redefinen sus roles en la actividad, los métodos y los modos de resolver satisfactoriamente el problema. Los diferentes puntos de vista o las confrontaciones que se producen en este proceso conducen a los sujetos a llegar a acuerdos que posibiliten avanzar hacia la resolución de la tarea. Para Y. Engeström (2015), estos eventos tienen como hecho incidental el aprendizaje. La interacción entre los sistemas de actividad que cada sujeto constituye produce una expansión del conocimiento. Así, el autor logra explicar cómo, qué y por qué aprendemos durante el desarrollo de una actividad cotidiana.

Las prácticas de enseñanza en la formación inicial no escapan a estos sistemas de actividad ni a los fenómenos de la interacción social. Sin embargo, a diferencia de la teoría propuesta por Engeström, las prácticas se encuentran dentro de un sistema didáctico, por tanto, tienen como objetivo principal el aprendizaje de un determinado contenido de saber. Como lo sostienen Pastré, Mayen y Vergnaud (2010), las prácticas en la formación profesional consisten en prolongar la actividad constructiva a partir de un análisis reflexivo de la acción misma con el fin de invertir la incidentalidad del aprendizaje de la actividad productiva ${ }^{1}$, y colocarlo como objeto principal de la actividad.

Si partimos de la propuesta de Engeström (2015), podemos ver que el sistema de actividad de las prácticas tiene un propósito principal: el aprendizaje de los saberes docentes o profesionales, y un objeto secundario: la resolución de la tarea, es decir, la experiencia de la acción pedagógica. La particularidad de este sistema es que se accede al objeto principal de la actividad mediante la obtención del objeto secundario: el saber, en cuanto objeto, es alcanzado (o construido) mediante la experimentación de las prácticas y la intervención de la interacción didáctica. En términos de mediación, según la definición de Vygotsky (1978), tanto la experiencia de las prácticas como la interacción representarían las herramientas o instrumentos que posibilitan la adquisición de los saberes.

La complejidad de las prácticas no puede, sin embargo, simplificarse a una relación causal entre sujeto-medios-objeto del sistema de actividad. La complejidad está en los medios mismos. La experiencia de la acción pedagógica y la interacción didáctica tienen, por naturaleza, una relación contradictoria. Por un lado, la acción implica un acto, un evento en el que un sujeto, en este caso un practicante, ejecuta una acción significativa, planificada e intencionada. La experiencia de la acción provoca un proceso de semiotización en el que el agente asigna un sentido a lo experimentado. Este proceso es individual y subjetivo, puesto que, como sostiene P. Ricoeur (1977), la acción le pertenece solo a él. Por otro lado, la interacción didáctica en esta situación de formación que tiene como intención última intervenir en ese proceso de asignación de sentido que le es ajeno. Los diferentes gestos de los profesores formadores, verbales y corporales, se orientan hacia los sentidos construidos por el practicante con la intención de ponerlos en cuestión, de confrontarlos a otros

\footnotetext{
Según la definición de los autores, la actividad productiva es la transformación de la realidad (material, social o simbólica) que se produce cuando un sujeto actúa. Pero, al transformar la realidad, el sujeto se transforma a sí mismo: es el lado de la actividad constructiva. La actividad constructiva representa el aprendizaje que el sujeto efectúa al realizar la actividad productiva.
} 
sentidos que emergen de las teorías científicas. La negociación verbal que se produce en esta interacción tiene como objetivo producir conflictos cognitivos en el practicante que lo lleven a resignificar su experiencia. Durante la interacción, los gestos didácticos del profesor permiten acompañar el nuevo proceso de asignación de sentidos, pero no pueden controlar la totalidad de las significaciones que el practicante realiza, puesto que esta depende de la situación biográfica determinada en el que este se encuentre. De esta relación conflictiva entre experiencia de la acción e interacción didáctica, el objeto principal de la actividad de la formación es alcanzado: la construcción de saberes profesionales.

\section{La configuración del repertorio didáctico, una estructura cognitiva interconectada que da sentido a la experiencia pedagógica}

La importancia de focalizarnos en el concepto de repertorio didáctico radica en que nos permite tratar los saberes docentes desde su complejidad, heterogeneidad e instrumentalidad. El concepto de repertorio en sí ya nos remite a la idea de conjunto de saberes y capacidades que los profesores disponen y utilizan en función de la situación de enseñanza en la que se encuentran. Esta fuente constituye la herramienta principal que es inherente a su actividad profesional.

Las autoras que introdujeron este concepto, F. Cicurel (2002) y M. Causa (2012), se interesaron por su construcción y su evolución en la formación docente inicial y continua. Ambas coinciden en que el repertorio didáctico se construye biográficamente a partir de una multiplicidad de fuentes, como la historia pedagógica, las teorías adquiridas durante la formación, el contacto con otros profesores y la experiencia en las prácticas efectivas. Este eclecticismo del repertorio les permite afirmar que se conforma a partir de un sistema de creencias, representaciones y saberes (Cambra, 2003). En nuestra investigación (Maldonado, 2019a, 2019b) nos hemos interesado por su conformación y organización y su incidencia en la asimilación de nuevos saberes durante la formación inicial.

En sintonía con los trabajos de Sendan y Roberts (1998), y Cabaroglu y Roberts (2000), hemos observado que el repertorio didáctico se compone de elementos proposicionales y elementos estructurales. Los proposicionales representan contenidos de saber; mientras que los estructurales, contenidos de sentido. Este último contiene la interpretación o el sentido subjetivo que el sujeto asocia a un saber y se construye históricamente a partir de la experiencia. Estos son los elementos que van a influir en la configuración del conjunto de la estructura. El contenido de sentido efectúa, al menos, dos tipos de acciones. Por un lado, otorga significatividad al contenido de saber y lo contextualiza dentro de la estructura. Por el otro, permite establecer una serie de relaciones o conexiones significativas con otras proposiciones cognitivas. Gracias a estas relaciones, los saberes se encuentran asociados entre sí. Estas asociaciones pueden referir a ideas de semejanza o contrariedad, de contigüidad en tiempo o lugar o de causa y efecto. Por ejemplo, los contenidos de saber sobre la lectura y la comprensión lectora pueden tener relaciones asociativas de contigüidad en tiempo y de causa y efecto. Estas conexiones significativas permitirían comprender la relación básica entre la lectura y la comprensión, por cuanto la segunda es consecuente de la primera. Otro ejemplo, que podría ilustrar la significatividad subjetiva de las asociaciones, es la conexión directa que establecen los saberes sobre la evaluación con la idea de control de la conducta. En términos didácticos, podríamos pensar que la evaluación estaría relacionada el aprendizaje, pero en los resultados de nuestra investigación observamos que los profesores asociaban causalmente la evaluación con el control de los alumnos. Los casos analizados utilizaban la evaluación para lograr que los alumnos cumplieran con determinadas tareas o actividades y no para obtener un indicador de lo aprendido.

La existencia de diferentes tipos de conexiones entre saberes nos permite hablar de un principio 
de asociación que regularía la estructuración cognitiva del repertorio didáctico. Este principio implicaría que los elementos o saberes establecen relaciones significativas entre ellos; así, crean redes que dan sentido a la existencia de dichos saberes dentro de la estructura. Las conexiones se extienden en dos direcciones. Por un lado, se dirigen a un elemento central, creando una red concéntrica con un núcleo que comparte un contenido de sentido con los elementos que lo rodean; por otro, los elementos periféricos establecen asociaciones entre sí, por más que no compartan el mismo núcleo. A estos dos fenómenos los hemos denominado principio de centralidad y principio de lateralidad. Por ejemplo, hemos observado que el saber sobre el control de la conducta representa un núcleo o un saber central dentro del repertorio didáctico. Alrededor de este se asocian una serie de contenidos de saber diversos: evaluación, preguntas de comprensión lectora, lectura en voz alta y corrección de las producciones escrita (principio de centralidad). El elemento preguntas de comprensión lectora, a su vez, establece relaciones asociativas de causalidad con interrelación textual y con preguntas de recuperación de saberes previos (principio de lateralidad).

Estos dos principios nos permiten explicar las relaciones entre saberes de una categoría a otra, creando así una imagen de red con múltiples nodos interconectados. Un elemento periférico, por tanto, puede tener una relación directa con otro de otra categoría, como se ve en la figura 1, en el que el elemento $b$ de la categoría $A$ establece una conexión directa con el elemento $c$ de la categoría $B$. Por otro lado, la relación entre el elemento a de la categoría $A$ con el elemento $f$ de la categoría $C$ se explica por el haz de familiaridad (Kleiber, 1990) que provoca la cadena de relaciones entre $a-b, b-c, c-d, d-e, e-f$. Esta relación indirecta es posible por el horizonte de familiaridad (Schütz, 2008) que cada elemento posee en relación con el conjunto de la estructura.

Ahora bien, la importancia de la configuración del repertorio didáctico en el proceso de aprendizaje en las prácticas de enseñanza se debe a dos motivos. Por un lado, es la fuente de saberes de referencia sobre la cual los practicantes se apoyan para realizar sus clases. Como sostiene P. Ricoeur (2000), al referirse a la capacidad de actuar de las personas, no existe acción sin saber. Los saberes que constituyen el repertorio didáctico representan la capacidad de actuar de los estudiantes y, como hemos mencionado, estos son heterogéneos. No todos los saberes han surgido de la formación académica, sino que son un entramado de saberes conformados históricamente de la experiencia de prácticas socioculturales diversas y de prácticas educativas. Según Cambra (2003, p. 213), la biografía pedagógica representa una de las fuentes más importantes en la construcción de modelos de enseñanza.

Siguiendo esta línea, vamos a considerar estas trayectorias personales como la mayor fuente de la que emergen los saberes que integran el repertorio

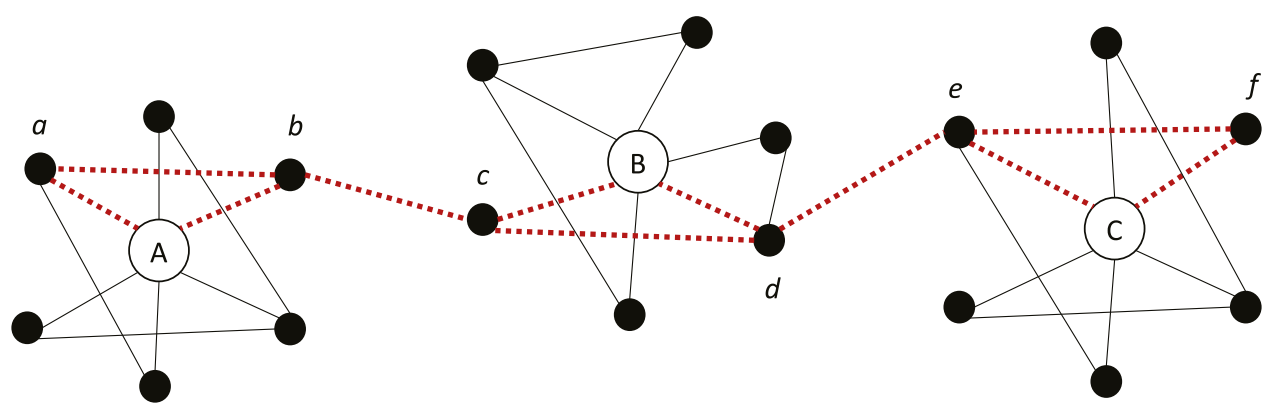

Figura 1. Principio de lateralidad

Fuente: Maldonado (2019a, p. 320). 
didáctico. Por otro lado, los tipos de conexiones entre los distintos elementos constitutivos de esta estructura cognitiva permiten interpretar y dar sentido a la experiencia de las prácticas y regulan el proceso de asimilación de nuevos saberes. Desde esta perspectiva, la configuración particular del repertorio puede significar el evento que permite la construcción de nuevos conocimientos $y$, al mismo tiempo, el punto de resistencia de su evolución.

La consideración de las implicancias de la configuración del repertorio didáctico en el proceso de las prácticas de enseñanza puede conducir a crear situaciones de enseñanza significativas que motiven a los practicantes a interrogarse sobre la naturaleza de sus saberes de referencia. La toma de conciencia del origen de los propios saberes lleva a modificar ciertos modelos de enseñanza asimilados o construidos a partir de la propia experiencia como aprendices.

\section{Metodología}

El estudio de caso puede ofrecernos indicios que nos permitan aproximarnos a una explicación y comprensión de la evolución del repertorio didáctico durante las prácticas de enseñanza y de los eventos que motivan o posibilitan dicha evolución. Por ello, nos hemos interesado en la experiencia de Laura, una estudiante del profesorado en Letras de la Universidad de Buenos Aires que realizó sus prácticas entre agosto y octubre de 2017, en un cuarto año del nivel secundario. Martina, la profesora formadora, realizó el seguimiento de las prácticas de Laura a través de la escritura de los guiones conjeturales y autorregistros, dispositivos de formación que se construyen de forma dialógica a partir de narraciones reflexivas (Bombini, 2012, 2018). En estos dispositivos encontramos interacciones verbales escritas cuya temática es la acción pedagógica. Laura y Martina hablan sobre las distintas problemáticas que se presentan en las prácticas, que refieren a la planificación de las clases y a los eventos sucedidos durante las clases dadas.

\section{Nuestra metodología de análisis}

Desde la perspectiva del análisis de los sistemas de actividad (R. Engeström, 1995; Y. Engeström, 2015), consideramos el proceso de las prácticas de enseñanza de Laura como el desarrollo de una actividad sociocultural, cuyo objeto es la experimentación de la acción pedagógica. Las interacciones entre practicante y formadora, por tanto, problematizan sobre ese objeto con el fin de llegar a acuerdos que conduzcan a una resolución satisfactoria para ambas de la tarea. Estas interacciones se producen desde el rol y lugar que cada una ocupa en la actividad: Laura, estudiante y profesora practicante; Martina, profesora experta. El desarrollo de la actividad y la resolución de la tarea provocan que el repertorio didáctico evolucione. Para observar estas transformaciones que se producen a nivel cognitivo, nos hemos focalizado en el análisis de las trazas de saberes y del proceso de enseñanza/aprendizaje que ambas dejaban en la configuración discursiva de sus intervenciones (Filliettaz y Bronckart, 2005; Filliettaz, 2018).

Nuestro corpus de datos está constituido por los dispositivos de formación, es decir, por los guiones conjeturales y por los autorregistros. Desde el punto de vista discursivo, siguiendo a Charaudeau (1992), estos documentos utilizan un modo enunciativo en su organización, por tanto, representan secuencias conversacionales escritas.

Debido a la naturaleza textual e interaccional de nuestros datos, la metodología utilizada se construye sobre la base del análisis del discurso en interacción (Kerbrat-Orecchioni, 2005). Mediante la utilización de categorías temáticas, gramaticales, pragmáticas, discursivas, contextuales y temporales abordamos la dimensión enunciativa, la construcción espacio-temporal del yo enunciativo; la dimensión epistémica, los saberes convocados en la realización de la actividad y que nos dan acceso al yo epistémico; la dimensión interactiva, la construcción discursiva en función de la relación con el interlocutor, y la dimensión temática, la construcción del contenido de la interacción (Palou y Fons, 
2010; Filliettaz, 2018). Partimos de la premisa de que, en la construcción del discurso interactivo, los hablantes dejan trazas de sus procesos semióticos, es decir, de los sentidos que asignan a los hechos o eventos a los que refieren. El método del análisis del discurso nos permite identificar esas trazas y obtener indicios que nos llevan a realizar una interpretación didáctica de la situación de formación. Para el análisis del discurso de la acción, seguimos el método hermenéutico propuesto por Ricoeur (1977, 2010), quien considera que es posible adentrarse al pensamiento de la acción a través de la configuración discursiva que los enunciadores efectúan.

La combinación de las diferentes dimensiones del discurso consideradas y los tipos de categorías de análisis utilizadas nos permiten acercarnos a nuestro corpus desde una perspectiva holística. En la interpretación de los datos, atendemos a eventos que suceden tanto a nivel macro como micro, y consideramos los procesos que autorregulan y circulan entre ambos niveles.

El procedimiento de análisis del nivel micro consiste en la identificación de categorías temáticas que refieren a contenidos de saber. Estas categorías están asociadas a un modo discursivo y a un grado de asertividad (Giammatteo et al., 2011). La determinación del modo y de la asertividad depende de la utilización de las modalidades enunciativas que, según Cervoni (1987), pueden manifestarse a nivel léxico (verbos o adverbios modales), morfológico (los modos y el aspecto verbal), sintáctico (tipos de estructuras, por ejemplo, las de tipo hipotéticas) o prosódico (el orden de las construcciones sintácticas). Luego, en el nivel macro de la interacción, observamos el comportamiento de esas unidades temáticas en la línea progresiva de la interacción. Utilizamos categorías temporales que nos permiten codificar las modificaciones o mutaciones de sentidos de los contenidos de saber y categorías pragmáticas y discursivas para identificar las relaciones causales de esas transformaciones con los eventos discursivos, es decir, aquellos enunciados que motivan o provocan un cambio de sentido en las categorías temáticas.
Los indicios que nos permiten observar la evolución del repertorio didáctico emergen de la progresividad temática de la interacción, puesto que las trazas de los cambios de sentidos que sufren los contenidos de saber se van sucediendo secuencialmente de un turno de palabra a otro. En cuanto a los indicios sobre las causas que motivan esta evolución, estos surgen de la interpretación de las categorías pragmáticas y discursivas del nivel interactivo. Las estrategias discursivas que cada enunciador utiliza para provocar cambios en el comportamiento del interlocutor mantienen una relación causal con los eventos cognitivos que se producen en la instancia de formación.

\section{Resultados y discusión}

\section{Los gestos discursivos en la creación de situaciones significativas y potenciales de aprendizaje}

A partir del concepto de posturas y gestos profesionales de profesores formadores propuesto por Jorro (2016) y del análisis de la dimensión interactiva y enunciativa del discurso, hemos identificado una serie de gestos discursivos recurrentes que Martina utiliza para crear situaciones significativas y potenciales de aprendizaje. Estos gestos representan un comportamiento discursivo determinado, cuyo fin es provocar una movilización cognitiva en el practicante que lo lleve a modificar sus prácticas $y$, en consecuencia, a una evolución de su repertorio didáctico. El aprendizaje, la asimilación de un contenido de saber dependerá de la capacidad del gesto de crear grados de significatividad para el estudiante.

El primer gesto discursivo que identificamos es la remarca positiva y negativa. Este tiene la intención de poner en evidencia o remarcar sentidos expresados por Laura y que representan, en cierta forma, el objeto de formación. Los referentes de estas remarcas pueden ser secuencias didácticas o actividades propuestas, reflexiones u opiniones sobre algún hecho de las prácticas, escenas o 
secuencias de acciones experimentadas durante las clases dadas.

Las remarcas positivas expresan un acuerdo o un grado de adhesión a un sentido. En algunos casos, se realizan algunas aclaraciones que modifican o añaden un nuevo sentido al objeto de la remarca, sin que esto signifique una oposición al sentido de base. Hemos encontrado en el discurso de Martina dos formas de estructuras semánticas que expresan una remarca positiva: "X es/está positivo/bien", "X significa que $\mathrm{P}$ ". Con la primera estructura, Martina valida o muestra su adhesión al pensamiento de Laura, dependiendo la naturaleza del referente.

Laura: Me cuentan que la hora que viene tienen evaluación de química, que por eso estaban "en otra cosa". [...] Sé que no van a leer nada en casa, así que voy a tener que pensar alguna estrategia para recuperar el sentido de ambas lecturas.

Martina: Punto clave, sí.

En este ejemplo, el referente de la remarca es la intención de una acción o actividad futura que Laura piensa realizar en la clase siguiente: "Voy a tener que pensar alguna estrategia para recuperar el sentido de ambas lecturas". Martina expresa que esta acción, el gesto de recuperar las lecturas, es un "punto clave" para la clase y, por tanto, es importante retener. La remarca nos permite inferir una adhesión total al sentido que Laura dé a la actividad de recuperación de lectura en esta situación. El sentido agregado sería el nivel de importancia de realizar tal actividad, "punto clave". Como vemos, Martina no aporta un nuevo contenido de saber de formación, simplemente, al validar la propuesta, refuerza un saber ya contenido en el repertorio didáctico de Laura.

El segundo tipo de estructura semántica de remarca positiva, " $\mathrm{X}$ significa que $\mathrm{P}$ ", consiste en la focalización de un evento y en una resignificación o una ampliación del sentido expresado. Por lo general, hemos identificado estas estructuras en los relatos de las clases dadas. En una escena de la tercera clase, Laura narra la secuencia didáctica de lectura de un texto literario y el debate ocurrido luego. En su relato, hace breves referencias al caos que generaban los alumnos durante el comentario de la lectura. En anteriores descripciones, ella había caracterizado al grupo de alumnos como tranquilo y ordenado para trabajar. Martina, al final del relato, con la intención de hacer una valoración general de lo ocurrido en la clase, vuelve sobre esta escena:

Martina: Rescato el despelote [desorden] en que se mueven ahora esos chicos modelo.

En esta remarca, Martina contrasta la imagen de los alumnos modelos, tranquilos, que Laura había descrito anteriormente, con el comportamiento en esta clase. El desorden en el que se realizó el debate no es un punto negativo para Martina, puesto que esto significó que los alumnos se interesaron y se involucraron en la actividad. Al topicalizar el verbo "rescato" en su enunciado, efectúa una resignificación de la imagen de los alumnos. A partir del contraste "alumnos modelos"/"alumnos desordenados", pone en valor la segunda imagen con el fin de modificar la representación negativa que Laura tiene respecto a este tipo de comportamiento. A pesar de haber un contraste, la remarca positiva consiste focalizarse sobre esta situación y darle un nuevo sentido, en este caso, positivo, a lo sucedido. La utilización de esta estructura semántica en el gesto de remarca positiva posibilita generar un aprendizaje a partir de una resignificación de lo experimentado. Mientras que, en un momento, el sentido que Laura asignaba a estas situaciones era negativo, luego de la remarca de Martina, este se vio modificado. Hemos observado que, en las clases posteriores, el caos o el desorden durante los debates eran provocados intencionalmente por ella. Sus comentarios al respecto eran del tipo "fue bueno", "hablaban mucho y así pudieron resolver el problema", "parece que les gustó el cuento porque discutieron ferozmente...".

Las remarcas negativas, a diferencia de las anteriores, se focalizan en situaciones determinadas 
con el fin de rechazar o invalidar un sentido expresado por la practicante y ofrecer una resignificación que se sitúa en un punto opuesto. En la segunda clase, Laura planifica una secuencia de lectura de una leyenda de un autor del Romanticismo español. Los alumnos ya conocen las características de este movimiento literario, puesto que era el tema de la clase anterior. Martina realiza una remarca negativa sobre la propuesta de actividad que sigue a la lectura:

Laura: La propuesta de esta clase es dedicarle el tiempo necesario a la lectura de la leyenda. Aproximadamente 30 minutos. Luego, les pregunto a los chicos si con lo que conocen de movimientos artísticos, pueden ubicarlo en alguno. [...]

Martina: ¿Por qué [harías esto]? ¡Listo, intento de no darles un prejuicio! Con esta pregunta, ¿qué creés que van a pensar? Estás dirigiendo la respuesta. Por otro lado, cuando leemos literatura, ¿enseguida la clasificamos? ¿Y [lo que dice Jean-Marie] Privat [sobre la lectura]? ¿Y [lo que estudiamos] de Michel de Certeau? ¡Habría que revisarlos!

Martina se focaliza sobre la segunda actividad, "les pregunto a los chicos si con lo que conocen de movimientos artísticos, pueden ubicarlo en alguno". La intención principal de sus enunciados es rechazar la secuencia propuesta. Con esta invalidación no solo se sitúa en la propuesta en sí, sino también en los sentidos o en los saberes sobre los que se basó Laura para elaborar la actividad. Si descomponemos lo que dice Martina en estos enunciados, nos encontramos con dos sentidos que se oponen o se contradicen a los inferidos de la actividad de Laura. Por un lado, la pertinencia de la pregunta. Para Martina, preguntar sobre la clasificación del texto leído en un movimiento literario puede resultar obvio para los alumnos, si el tema que se estudia es justamente el Romanticismo. Por tanto, para ella no es una pregunta pertinente y no representa ningún desafío cognitivo, puesto que los alumnos pueden llegar a responder a partir de un simple proceso de razonamiento inferencial basado en los indicios del contexto y no porque efectivamente conozcan las características del movimiento literario. El segundo de esto es el sentido de la secuencia lectura más la clasificación del texto. Este tipo de actividad va en dirección opuesta a los saberes teóricos de referencia sobre la didáctica de la lectura. Por ello, reforzar este punto cita a dos autores, Privat y Certeau. El aprendizaje de los contenidos de saber de la formación, en este caso, se produciría por la punición de la experiencia y una resignificación hacia sentidos opuestos. Es decir, el hecho de invalidar la actividad o el gesto, implica que tal o cual acción no es correcta y, en consecuencia, no se debe realizar.

El segundo tipo de gesto discursivo que utiliza Martina es la creación de situaciones críticas que problematizan una acción o un sentido expresado por Laura. La finalidad de este gesto es lograr que la practicante modifique, ya sea su actividad planificada, ya sea su conducto o los sentidos construidos. Estos objetivos se logran mediante dos tipos de acciones: a) la puesta en evidencia de ciertas contradicciones inferidas del discurso, b) la introducción de una nueva evidencia o no considerada por la practicante. Cualquiera que fuera el caso, lo que permite el movimiento cognitivo es la toma de consciencia de la existencia de ideas o sentidos opuestos.

Dos tipos de recursos discursivos, según hemos podido observar, permiten la creación de situaciones críticas: el discurso hipotético y las preguntas reflexivas. El primero podríamos representarlo mediante el enunciado "qué pasaría si...". Estas estructuras típicas expresan una situación hipotética que a la vez es probable o posible que suceda y contraria a la situación imaginada por el practicante. Mediante este recurso, la profesora formadora induce al practicante a imaginar un comportamiento determinado o una secuencia de acciones diferente al que planificó. Por ejemplo:

Martina: En caso de que no surgiera... ¿Cómo imaginás? ¿Lo repondrías vos? O, ¿intentaríamos 
seguir buscando que aparezca esta cuestión leyendo por ejemplo al Todorov que sigue?

Martina: ¿Y si alguno quisiera intercambiar [el texto] con un compañero?

Martina: [...] Y sobre la tarea que quedó según tu segundo autorregistro... ¿Qué pasaría si no la realizan? ¿Cómo se retomaría? ¿De qué manera?

Martina: En caso de realizarse lectura grupal, ¿Cómo imaginás que se irá dando el encuentro para hacer más accesible el relato?

Martina: ¿Y si no los leyeron todos?

Como se puede observar, en todos estos ejemplos hay una pregunta del tipo "qué pasaría si...". Al crear una situación hipotética, Martina introduce una contraevidencia o una información desconocida o no tenida en cuenta que modifica la situación imaginada por Laura. Esta modificación provoca la necesidad de cambiar de estrategia o replanificar la actividad, como se puede ver en la respuesta a la pregunta del ejemplo (6):

Laura: En caso de que el cuento no cumpla para nada con el género, les daré la posibilidad de reescribir aquello que no encuadra [...]. Sobre el escrito de "El corazón delator", la voy a retomar oralmente en esta clase, al principio, para recuperar lo conceptual sobre el fantástico.

Las preguntas reflexivas se caracterizan por tomar la forma discursiva de una pregunta directa pero cuya intención no es solicitar algo sino inducir al practicante a tomar una postura reflexiva sobre un tema concreto. En la siguiente secuencia de interacción, Laura coloca entre comillas una expresión, sin hacer referencia a por qué las utiliza.

Laura: [...] Muy pocos habían leído Axolotl y Casa tomada, y quienes lo habían hecho tenían dudas... pero les pedí que las guardaran para el miércoles, para insistirles a quienes no habían leído que lo hicieran, ya que "entran en la evaluación".

Martina: ¿Las comillas? ¿Qué pensabas cuando las ponías en el registro?
Laura: Creo que es el eterno cavilar entre intentar motivarlos a que se acerquen a la lectura y utilizar las formalidades para que, aquellos que casi nunca acceden a participar, repentinamente se "despabilen".

Las preguntas de Martina sobre las comillas Ilevaron a Laura a adoptar una postura reflexiva sobre su discurso y sobre su pensamiento. Esto permitió que exteriorizara ciertas problemáticas o tensiones que ella experimenta respecto a la enseñanza de la literatura y la motivación de los alumnos. A diferencia del recurso de las situaciones hipotéticas, las preguntas reflexivas no oponen sentidos o no expresan una contradicción, simplemente problematizan una situación determinada que lleva a la estudiante a exteriorizar discursivamente una contradicción o un conflicto cognitivo, como se ve en el ejemplo citado. A partir de esta exteriorización, el formador puede orientar a la estudiante a resolver el conflicto.

El tercer gesto discursivo utilizado por Martina es la institucionalización. Este recurso, próximo a la remarca positiva, rescata lo dicho por Laura y formaliza el contenido de saber. Los enunciados suelen tomar la forma de declaraciones afirmativas que utilizan un presente gnómico. Esto les da el carácter de verdades categóricas y atemporales:

Martina: [...] Esto que decís al final, tenelo en cuenta para otros modos de evaluación en los alumnos que no se sientan puestos a prueba. Insisto con la escritura de ficción porque, además de estudiar la teoría, lo hago con mis alumnos de secundaria. Sacás mejores resultados de los chicos y no sienten esa presión de tener que estudiar el tema 1, el tema 2, que es inevitable.

Martina: [Esto que contás me recuerda a] lo que charlábamos de lo que se copia, el objetivo de hacer dictados es de inflar las carpetas... Para seguir pensando en la construcción de la escuela en el imaginario.

En estos dos ejemplos, Martina realiza dos gestos de institucionalización, uno refiere a la 
utilización de la escritura de ficción como instrumento de evaluación del aprendizaje, y la otra, a la función real del dictado en clase. La institucionalización, como todos los otros gestos, aporta nuevos sentidos a los eventos descritos por Laura, pero a diferencia del resto, son enunciados realizados desde una postura de especialista, de conocedora de la teoría y de la experiencia, no desde una interpretación subjetiva: "Insisto con la escritura de ficción porque, además de estudiar la teoría, lo hago con mis alumnos de secundaria". El carácter de verdad absoluta e incuestionable del enunciado lo toma de esta condición. Esto explica el hecho de que no encontráramos escenas de confrontación o de cuestionamiento en las interacciones que seguían a estos gestos. Laura acepta sin cuestionar el contenido de estos enunciados y lo incorpora a su repertorio didáctico. En la última clase, el tipo de evaluación propuesta está elaborado a partir del concepto de escritura de ficción, tal y como lo había mencionado Martina. La reflexión que realiza al respecto da cuenta de que este saber ha sido incorporado a su repertorio:

Laura: $[. .$.$] para escribir el cuento tuvieron que$ elegir primero el tipo de narrador y luego tener en cuenta las características del género. La mayoría de las producciones respetaron muy bien estas consignas. ¡Los chicos prestaron atención en las clases y aprendieron!

Por último, otro gesto identificado es la instrucción, que se manifiesta discursivamente en una orden o una sugerencia. Este recurso aparece normalmente en las planificaciones cuando Laura, luego de varias reformulaciones, no logra proponer una actividad didáctica con una metodología de enseñanza adecuada. Según la frecuencia de aparición, nuestro análisis nos permitió ver que no es el tipo de gesto más utilizado puesto que el contexto o la situación comunicativa en el que emerge solo se da en tres momentos. Otras de las características que hemos podido observar es que no representa un grado significativamente importante dentro del proceso de aprendizaje de Laura. La asimetría jerárquica y la relación de poder son los factores que nos permiten explicar las razones de por qué Laura lleva a cabo las ordenes o las sugerencias de la formadora. La aceptación de ejecutar una acción sugerida u ordenada no significa que haya asimilado un contenido de saber puesto que la significatividad está focalizada en la intención de cumplir una orden más que en el sentido de la acción misma. Hemos observado que la significatividad de la acción es encontrada durante o luego del acto. Una de las sugerencias que Martina le había hecho a Laura, para su tercera clase, era trabajar la teoría no a partir de una exposición sino a partir de preguntas que buscaran relacionar lo nuevo con los conocimientos que los alumnos ya tenían. Laura modificó su planificación e hizo lo que su formadora le pedía. Sin embargo, no fue hasta la quinta clase que logró encontrar sentido a esta acción luego de haber experimentado y haber obtenido resultados positivos:

Laura: Ahora encuentro sentido a esto que siempre decís de desarrollar la teoría a partir de preguntas. Me he dado cuenta de que los alumnos conocen mucho y que, si les comienzo a preguntar, las cosas van saliendo solas. Aprendieron más en esta clase sobre los tipos de narradores que en las anteriores.

\section{Conclusiones}

Como hemos observado, el proceso de aprendizaje y asimilación de un contenido de saber de la formación durante la interacción verbal entre Martina y Laura se ve condicionado por el tipo de gesto discursivo que Martina utiliza para crear situaciones significativas y potenciales de aprendizaje. La configuración particular del repertorio didáctico de Laura y su situación biográfica determinada inciden en la asignación de sentidos a la experiencia de sus prácticas. Hemos encontrado escenas en el discurso de Laura en el que los sentidos se contradecían entre sí o se posicionaban en 
un punto opuesto a los modelos de enseñanza actuales. Estas contradicciones no eran evidentes ni significativas para ella. Desde el rol de formadora, Martina ponía en evidencia estos sentidos opuestos, pero esto no producía necesariamente un grado de significatividad. La significatividad dependió de las estrategias didácticas y de los gestos pedagógicos para generar situación que conducían a Laura a encontrar por sí sola la contradicción.

La creación de situaciones hipotéticas podría representar una estrategia potente a la hora de movilizar determinados saberes de los practicantes. Este recurso, por un lado, ofrece nuevos indicios o evidencias que permiten proyectar una situación diferente a la imaginada por el practicante; por el otro, detrás de esos indicios ofrecidos se encuentra un contenido de saber de la formación. Al apropiarse de las nuevas evidencias contenidas en el "qué pasaría si...", el practicante asume posturas determinadas que le permiten reaccionar sobre esa nueva situación hipotética. En ese cambio de posturas quedarían evidenciadas las contradicciones y la significatividad se construiría de los nuevos gestos asumidos. Desde este punto de vista, el repertorio didáctico evolucionaría no solo por la asimilación o aprendizaje de nuevas situaciones posibles sino por la búsqueda de nuevas formas de actuar sobre esas situaciones imaginarias.

La interacción didáctica, en este caso al estar vehiculizada únicamente por la escritura de este dispositivo de formación, otorga al discurso un rol determinante en el proceso de enseñanza/aprendizaje. Si en lugar de utilizar el gesto discursivo de situación hipotética, Martina se valiera de la instrucción para provocar la movilización cognitiva, podríamos ver que Laura se limitaría a cumplir las órdenes sin la necesidad de que estas acciones sean necesariamente significativas para ella. El aprendizaje y la evolución del repertorio didáctico estarían, por tanto, condicionado por la semiotización que Laura realice de la experimentación de la acción y no como consecuencia de una resignificación orientada e intencionada de una interacción didáctica.

\section{Reconocimientos}

Este artículo presenta uno de los resultados de la investigación "La circulación de saberes en la formación inicial del profesorado en Lengua y la construcción del repertorio didáctico", el cual se inscribe en el programa de Doctorado en Didáctica de las Ciencias, las Lenguas, las Artes y las Humanidades, de la Universitat de Barcelona, España.

\section{Referencias bibliográficas}

Bombini, G. (2012). Escribir la metamorfosis. Escritura y formación docente. Buenos Aires, Argentina: El Hacedor.

Bombini, G. (2018). Didáctica de la lengua y la literatura: entre la intervención y la investigación. Bellaterra Journal of Teaching \& Learning Language \& Literature, 11(4), 5-20. DOI: https://doi. org/10.5565/rev/jtl3.784

Cabaroglu, N. y Roberts, J. (2000). Development in studentteachers'pre-existingbeliefsduringa1-YearPGCE programme. System, 28(3), 387-402.

Cambra Giné, M. (2003). Une approche ethografique de la classe de lengue. París, Francia: Didier.

Cambra Giné, M. y Palou, J. (2007). Creencias, representaciones y saberes de los profesores de lenguas en las nuevas situaciones plurilingües escolares de Cataluña, Cultura y Educación, 19(2), 149-163, DOI: 10.1174/113564007780961651

Causa, M. (2012). Le répertoire didactique: une notion complexe. En M. Causa (ed.), Formation initiale et profils d'enseignants de langues. Enjeux et questionnements (pp. 15-72). Bruselas, Bélgica: De Boeck Supérieur.

Cervoni, J. (1987). L'énonciation. París, Francia: PUF.

Charaudeau, P. (1992). Grammaire du sens et de l'expression. París, Francia: Hachette.

Cicurel, F. (2002). La classe de langue, un lieu ordinaire, une interaction complexe. AILE, 16, 145164. Recuperado de http://journals.openedition. org/aile/801

Cicurel, F. (2016). Reconfigurer l'action enseignante pour la (re) découvrir: traces du répertoire 
didactique évolutif. Phronesis, 5(3), 16-27. DOI: https://doi.org/10.7202/1039083ar

Edelstain, G y Coria, A. (1995). Imágenes e imaginación. Inicio a la docencia. Buenos Aires, Argentina: Kapelusz.

Engeström, R. (1995). Voice as communicative action. Mind Culture and Activity, 2(3),192-215. DOI: 10.1080/10749039509524699

Engeström, Y. (2015). Learning by expanding an activity-theoretical approach to developmental research. 2a. ed. Cambridge, EE. UU.: Cambridge University Press.

Fillietaz, L. (2018). Interactions verbales et recherche en éducation. Principes, méthodes et outils d'analyse. Ginebra: Université de Genève

Filliettaz, L. y Bronckart, J.P. (eds) (2005). L'analyse des actions et des discours en situation de travail. Concepts, méthodes et applications. Lauvainla-Neuve: Peeters.

Giammatteo, M. et al. (2011). Dos dominios en intersección: habitualidad y posibilidad. Su manifestación en las perífrasis verbales. En J. Cuartero, L. García Fernández y C. Sinner (eds.), Estudios sobre perífrasis y aspecto (pp. 139-157). Múnich: Peniope.

Goffman, E. (1973). La mise en scène de la vie quotidienne. Tome 1. La présentation de soi. París, Francia: Minuit.

Jorro, A. (2016). Postures et gestes professionnels de formateurs dans l'accompagnement professionnel d'enseignants du premier degré. eJRIEPS, 38, 114-132.

Kerbrat-Orecchioni, C. (2005). Le discours en interaction. París, Francia: Armand Colin.

Kleiber, G. (1990). La Sémantique du Prototype. París Francia: PUF.

Leleu-Merviel, S. (2013). Traces, information et construits de sens. Déploiement de la trace visuelle de la rétention indicielle à l'écriture. Intellectica, Revue de l'Association pour la Recherche Cognitive, 59(2013/1), 65-88.

Maldonado, M. (2019a). La circulación de saberes en la formación inicial del profesorado de lengua y la construcción del repertorio didáctico. (Tesis doctoral). Universitat de Barcelona, Barcelona.

Maldonado, M. (2019b). Los saberes docentes en la formación inicial. Revista Alquimia Educativa 5(2), 31-61. Recuperado de https://unca-ar.academia. edu/RevistaAlquimiaEducativa

Palou, J. y Fons, M. (2010). Metacognició i relats de vida linguîstica en els processos de formació del professorat. Anàlisi del relat d'una alumna. En Multilinguiisme i pràctica educativa (pp. 255-260). Girona: Universitat de Girona.

Pastré, P., Mayen, P. y Vergnaud, G. (2010). La didactique professionnelle, Revue Française de Pédagogie, 154. 154-198.DOI: https://doi.org/10.4000/ rfp. 157

Perrenoud, Ph. (2012). Le travail sur I'habitus dans la formation des enseignants. Analyse des pratiques et prise de conscience. En L. Paquay et al., Former des enseignants professionnels (pp. 211-237). Bruselas, Bélgica: De Boeck Supérieur. DOI: 10.3917/dbu. paqua.2012.01.0211

Ricoeur, P. (1977). La sémantique de l'action. París, Francia: CNRS.

Ricoeur, P. (2000). Del texto a la acción. Ensayos de Hermenéurica II. México: Fondo de Cultura Económica.

Ricoeur, P. (2010). Écrits et conférences 2. Hermenéutique. París, Francia: Seuil

Schütz, A. (2008). Le chercheur et le quotidien. París, Francia: Klincksieck.

Sendan, F. y Roberts, J. (1998). Orhan: a case study in the development of a student teacher's personal theories. Teachers and Teaching, 4(2), 229-244, DOI: 10.1080/1354060980040203

Stake, R.S (1999). Investigación con estudio de casos. Madrid, España: Morata.

Vygotsky, L.S. (1978). Mind in Society, the Development of Higher Psychological Processes. Cambridge: Harvard University Press Cambridge. 